\title{
Thermal analysis of electric vehicle DC charging pile power module based on two-dimensional ordered porous structure radiator
}

\author{
Ying Han ${ }^{1, *}$, Zhi-jun $\mathrm{Liu}^{2, * *}$, Chun-guang $\mathrm{Hou}^{3}$, Yun-dong Cao ${ }^{3}$, Li-rong Zhai ${ }^{4}$ \\ ${ }^{1}$ School of Electrical Engineering, Shenyang University of Technology, Tiexi District, Shenyang, Liaoning, China \\ ${ }^{2}$ School of Electrical Engineering, Shenyang University of Technology, Tiexi District, Shenyang, Liaoning, China \\ ${ }^{3}$ School of Electrical Engineering, Shenyang University of Technology, Tiexi District, Shenyang, Liaoning, China \\ ${ }^{4}$ School of Light Industry, Liaoning University, Huanggu District District, Shenyang, Liaoning, China
}

\begin{abstract}
In order to improve the heat dissipation performance and study the factors affecting the heat dissipation effect of a two-dimensional ordered porous structure, a thermal analysis of the radiator in the power module of a DC charging pile was carried out. Based on the thermal analysis of the grid-type radiator, the square-hole radiator is subjected to a thermal analysis, the heat dissipation performance of the two radiators is compared, and the factors affecting the heat dissipation effect of the square-hole radiator are explored. Research shows that the heat dissipation effect of the square-hole radiator, which represents a twodimensional ordered porous structure, is better than that of the grid-type radiator. The factors affecting the heat dissipation effect of the square-hole radiator are mainly related to the height of the radiator and are positively related to the heat dissipation effect of the radiator.
\end{abstract}

\section{Introduction}

Porous materials consist of a continuous solid phase that forms the basic framework of the material itself and a fluid phase that forms voids. Porous materials are divided into two categories, one is disordered porous materials with irregular distribution of pores, and the other is ordered porous materials with regular distribution of pores. Among them, the twodimensional ordered porous structure is often used as the basis for the study of more complex structures due to its regular arrangement. Due to the higher specific surface area, it has more advantages than traditional grid radiators in enhancing heat transfer.

In the 1990s, Bejan Adrian[1]proved that when fluid flows through a certain length of twodimensional ordered porous material, it produced a pressure drop and affect heat transfer. $\mathrm{Gu}$ et al.[2]constructed several typical two-dimensional porous materials such as regular triangles, regular quadrilaterals, regular hexagonal holes and studied the advantages and disadvantages of their respective performances.Wen et al.[3]conducted experimental evaluations on material samples with different thermal conductivity based on $\mathrm{Gu}$ and added rhombic and trapezoidal structures. The results showed that the pressure drop mainly depends on the porosity and pore shape, and the thermal conductivity is also related to the thermal conductivity of the wall material. The coefficient is related.In recent years, many scholars have carried out research work on the analysis and design of two-dimensional porous materials with both loadbearing and heat-dissipating properties. Liang Xiaolin et al.[4]used porous materials on the radiator and compared them with traditional finned radiators.Xia et al.[5]design the hole shape as a curved-edge figure, and conduct thermal analysis on curved-sided triangles, curved-sided quadrilaterals, and ellipses.

\section{Theory and methods}

Any flow and heat transfer problem must follow the law of conservation of mass, momentum, and energy, and the governing equations are as follows:

(1) Mass conservation equation:

$$
\frac{\partial \rho}{\partial t}+\nabla \cdot(\rho U)=0
$$

(2) Momentum conservation equation:

$$
\nabla \cdot(u U)=\nabla \cdot(\mu \nabla u)-\frac{1}{\rho} \frac{\partial p}{\partial x}
$$

*email: hany_dq@sut.edu.cn,**email: nasionliu@163.com, Corresponding Author:**email:nasionliu@163.com 


$$
\begin{gathered}
\nabla \cdot(v U)=\nabla \cdot(\mu \nabla v)-\frac{1}{\rho} \frac{\partial p}{\partial y} \\
\nabla \cdot(w U)=\nabla \cdot(\mu \nabla w)-\frac{1}{\rho} \frac{\partial p}{\partial z}
\end{gathered}
$$

(3) Energy conservation equation:

$$
\nabla \cdot(U T)=\nabla \cdot\left(\frac{\lambda_{g}}{\rho C} \nabla T\right)
$$

(4) The equation of heat conduction between solids:

$$
\nabla \cdot\left(\lambda_{s} \nabla T\right)=0
$$

(5) The convection heat transfer equation between solid gas:

$$
\nabla \cdot\left(\lambda_{s l} \nabla T\right)=0
$$

Where: ${ }^{U}$ is the velocity vector of the fluid. in the space $x y, z$ component in the direction is $u, v$, $w{ }^{T}$ are the temperature. ${ }^{p}, \rho, \mu, C$ are the pressure, density, viscosity coefficient and specific heat capacity of the fluid respectively. ${ }^{\lambda_{g}},{ }^{\lambda_{s}},{ }^{\lambda} s$ are the thermal conductivity between fluid, solid, and solid gasare between the fluid and the solid.

\section{Simulation research results}

According to the current radiator used by the power module in an electric vehicle DC charging pile and the size of the installation space.Keeping the boundary conditions consistent, the power module runs for 10 minutes, and the simulation results are shown in Figures 1 and 2. The maximum surface temperature of the grid radiator is $70^{\circ} \mathrm{C}$, the maximum surface temperature of the square hole radiator is $65.6^{\circ} \mathrm{C}$. The surface temperature is higher than that of the square hole radiator, indicating that the heat dissipation effect of the square hole radiator is better than that of the grid radiator.
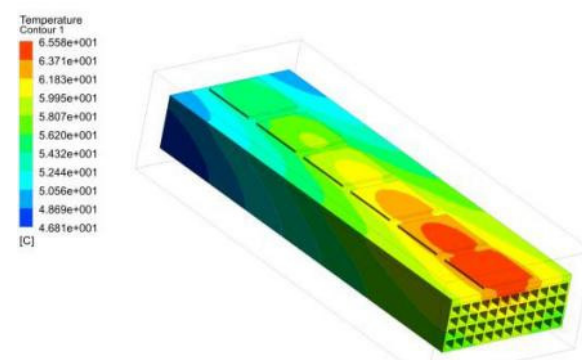

Figure 1 Temperature cloud diagram of grid radiator
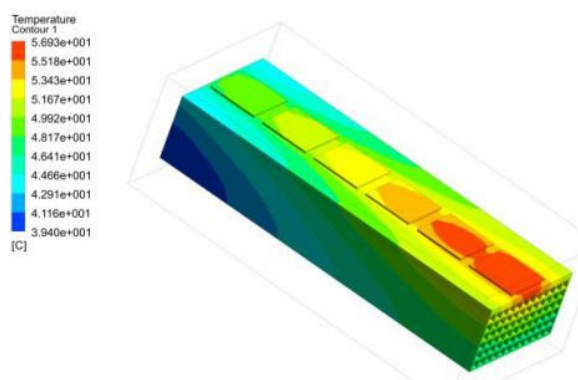

$\tau$

Figure 2 Temperature cloud diagram of square hole radiator

\subsection{Factors affecting square hole radiators}

There are many factors that affect the square hole radiator and the main factor is radiator height.Keep other factors unchanged, set the height of the radiator to 6 layers, and the other dimensions remain unchanged. The simulation results are shown in the figure 3 .
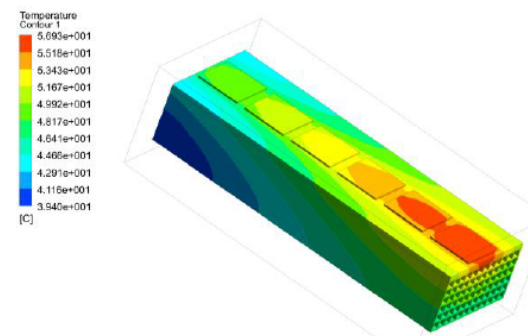

ז

Figure 3 6-layer square hole radiator temperature cloud map

The maximum surface temperature of the 6layer square hole radiator is $56.9^{\circ} \mathrm{C}$. It shows that when other factors are the same, the greater the height of the radiator, the better the heat dissipation effect. Add three sets of control experiments, the number of square hole layers are 7,8 , and 9 respectively. The relationship is shown in the figure 4.

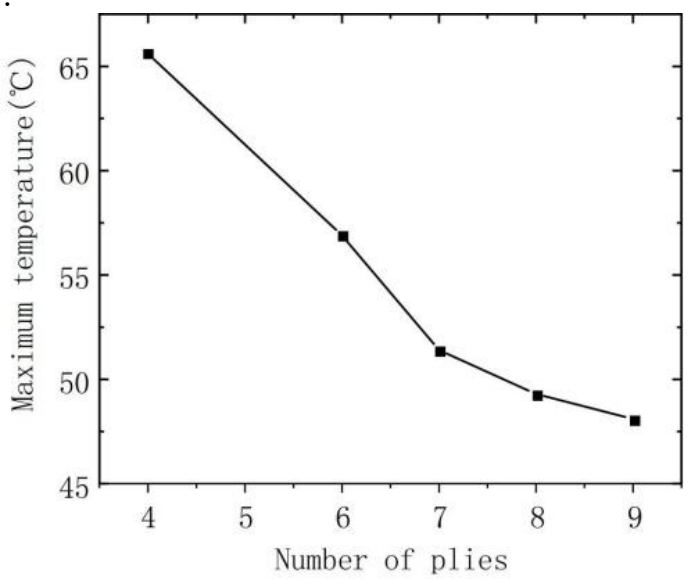

Figure 4 The relationship between the maximum surface temperature and the number of plies 


\section{Conclusions}

(1) When other factors are the same, the square hole radiator emits more heat than the common grid-type radiator through forced air cooling in the same time, and the heat dissipation effect is better.

(2) The main factors affecting the heat dissipation effect of the square hole radiator are the fluid velocity and the height of the square hole radiator. The heat dissipation effect is positively related to the above three factors.

\section{Acknowledgments}

1. Liaoning Province Natural Science Foundation Project no. 2019-MS-249.

2. National Natural Science Foundation of China no. 51871220 .

\section{References}

1. Bejan Adrian.Heat Transfer. New York: John Wiley \& Sons, Inc., 1993.

2. S. Gu, T.J. Lu, A.G. Evans. On the design of twodimensional cellular metals for combined heat dissipation and structural load capacity[J]. International Journal of Heat and Mass Transfer, 2001,44(11).

3. T. Wen, J. Tian, T.J. Lu, D.T. Queheillalt, H.N.G. Wadley. Forced convection in metallic honeycomb structures[J]. International Journal of Heat and Mass Transfer, 2006, 49(19).

4. Liang Xiaolin, Ding Li, Cao Hailin. Thermal analysis and structural optimization of two-dimensional porous material heat $\sin \mathrm{k}[\mathrm{J}]$. Electronic Mechanical Engineering, 2018, 34(04): 23-26.

5. Zhengnan Xia,Keyong Wang,Fengyan Ge. Special hole elements for simulating the heat conduction in two-dimensional cellular materials[J]. Composite Structures, 2020, 246. 